Proceeding Series of the Brazilian Society of Computational and Applied Mathematics

\title{
Sistema de Controle de Atitude Proposto para a Missão Espacial SERPENS II
}

Antonio Gil Vicente de Brum ${ }^{1}$

Centro de Engenharia, Modelagem e Ciências Sociais Aplicadas, UFABC, Santo André, SP

Leandro Baroni ${ }^{2}$

Centro de Engenharia, Modelagem e Ciências Sociais Aplicadas, UFABC, Santo André, SP

André Luís da Silva

Coordenadoria Acadêmica de Cachoeira do Sul, UFSM, Santa Maria, RS

Cristiano Fiorilo de Melo ${ }^{4}$

Escola de Engenharia, Dep ${ }^{\text {to }}$ de Engenharia Mecânica, UFMG, Belo Horizonte, MG

Eduardo dos Santos Ferreira

Centro de Engenharia, Modelagem e Ciências Sociais Aplicadas, UFABC, Santo André, SP

Maria Cecília Zanardi ${ }^{6}$

Centro de Engenharia, Modelagem e Ciências Sociais Aplicadas, UFABC, Santo André, SP

Departamento de Matemática, Unesp, Guaratinguetá, SP

Claudia Celeste Celestino

Centro de Engenharia, Modelagem e Ciências Sociais Aplicadas, UFABC, Santo André, SP

Maria Cecilia Pereira

Escola de Engenharia, Dep ${ }^{\text {to }}$ de Engenharia Mecânica, UFMG, Belo Horizonte, MG

Anderson W. Spengler ${ }^{9}$

Departamento de Engenharia Aeroespacial, UFSC, Florianópolis, SC

\footnotetext{
1 antonio.brum@ufabc.edu.br

2 leandro.baroni@ufabc.edu.br

3 taurarm@gmail.com

4 cristiano.fiorilo@demec.ufmg.br

5 eduardo.ferreira@ufabc.edu.br

6 cecilia.zanardi@ufabc.edu.br, cecilia@feg.unesp.br

7 claudia.celeste@ufabc.edu.br

8 cecilia@demec.ufmg.br

9 andersonws@gmail.com
} 
Resumo. A missão SERPENS envolve pesquisas e desenvolvimentos na área de nanossatélites e pertence ao conjunto de atividades conjuntas realizadas por universidades brasileiras no âmbito das atividades espaciais coordenadas pela Agência Espacial Brasileira - AEB. A missão SERPENS I, primeira da série, tem lançamento previsto para agosto de 2015. A missão SERPENS II encontra-se correntemente em projeto. Este trabalho trata da missão SERPENS II e descreve as atividades ligadas ao projeto do Sistema de Determinação e Controle de Atitude (ADCS) do CubeSat 3U SERPENS II. A missão e os respectivos requisitos em atitude são descritos. Uma proposta de ADCS adequada ao cumprimento dos objetivos da missão é apresentada. A modelagem do satélite e sua dinâmica de atitude são efetuadas, em função do ADCS proposto (hardware e software), e os resultados obtidos referentes ao primeiro modo de controle (descapotamento) são apresentados e considerados satisfatórios, corroborando a proposta inicial.

Palavras-chave. Missão espacial SERPENS, nanossatélite, CubeSat, controle de atitude, modelagem e simulação.

\section{Introdução}

Este trabalho está contido dentro do escopo do Sistema Espacial para Realização de Pesquisa e Experimentos com NanosSatélites (SERPENS). O projeto SERPENS, iniciado em agosto de 2013, teve sua estruturação inicial realizada pela Agência Espacial Brasileira (AEB) em conjunto com o curso de Engenharia Aeroespacial (EA) da Universidade de Brasília (UnB). O lançamento oficial do programa ocorreu em dezembro de 2013, onde foi constituído um consórcio para condução do mesmo, formado pelos cursos de EA das universidades: UnB, Universidade Federal de Santa Catarina (UFSC), Universidade Federal do ABC (UFABC) e Universidade Federal de Minas Gerais (UFMG); bem como o centro de referência em sistemas embarcados e aeroespaciais (CRSEA) do Instituto Federal Fluminense (IFF). Além de parceiros internacionais: Universidade de Vigo (UVigo) da Espanha, Università di Roma Sapienza da Itália, Morehead State University e California Polytechnic State University dos Estados Unidos. Contando também com a colaboração de institutos nacionais: Centro Regional Norte (CRN) do Instituto Nacional de Pesquisas Espaciais (INPE), em Natal/RN, e Laboratório de Sistemas Integráveis Tecnológico (LSITec) de São Paulo. O consórcio como um todo é gerenciado pela AEB.

Os objetivos principais do programa SERPENS são: promover e consolidar os novos cursos de EA brasileiros; qualificar docentes e pesquisadores vinculados aos cursos de EA do Brasil bem como de outros centros, como o IFF; envolver e capacitar estudantes e jovens profissionais em um projeto real de desenvolvimento de tecnologia espacial.

A injeção em órbita do satélite SERPENS I está prevista para outubro de 2015, por meio do sistema Kibo da Agência Espacial Japonesa (JAXA) a bordo da estação espacial internacional (ISS). O satélite começou a ser integrado em fevereiro de 2015 no Laboratório de Integração e Testes (LIT) do INPE, seu modelo de voo já foi aprovado, em conformidade com requisitos técnicos da JAXA. O equipamento também foi aprovado na Revisão de Segurança de Projeto (SAR, na sigla em inglês), realizada no Japão.

Neste cenário, os quatro cursos de EA das universidades participantes do SERPENS: UnB, UFSC, UFMG e UFABC, estão propondo uma segunda missão dentro do projeto SERPENS, denominada SERPENS II. A mesma encontra-se em fase de estruturação, com elaboração de uma proposta a ser submetida a órgãos de fomento. Este artigo trata do desenvolvimento do Sistema de Determinação e Controle de Atitude (ADCS) para o nanossatélite SERPENS II. 


\section{A Missão SERPENS II}

Assim como no projeto anterior, o nanossatélite proposto na missão SERPENS II é um CubeSat 3U, cuja órbita, inicialmente, será circular e terá aproximadamente $400 \mathrm{~km}$ de altitude, com inclinação entre $45^{\circ}$ e $60^{\circ}$. Neste tipo de órbita, os principais momentos perturbativos agindo no veículo são devido ao gradiente de gravidade, arrasto aerodinâmico, pressão de radiação e campos magnéticos residuais (por causa da eletrônica de bordo). A referência [7] quantifica estes torques como sendo da ordem de $10^{-8} \mathrm{~N}$.m para uma órbita de $600 \mathrm{~km}$.

A missão possuirá instrumentação para realização de três experimentos, descritos a seguir. A coordenação do projeto do ADCS é de responsabilidade de pesquisadores da UFABC, com colaboração da UFMG e UFSC.

Os requisitos em atitude para o SERPENS II se devem principalmente aos experimentos a ser realizados: 1) detecção de raios gama e X; 2) detecção da anomalia magnética do Atlântico sul (AMAS); 3) teste de um propulsor pulsado de plasma (PPT).

Requisitos em atitude para os experimentos 1 e 2 (fase 1) - Nesta etapa, sob o aspecto de transmissão de dados, qualquer atitude é aceita, devido ao uso de antenas omnidirecionais. Para medição da AMAS, não há requisitos rigorosos na atitude. No entanto, os sensores de raios X e gama não podem ser apontados para a Terra ou outras fontes brilhantes (Sol e Lua). Um modo de estabilização por rotação (spin) deverá ser implementado nesta etapa da missão, com eixo de rotação dependente da direção de apontamento dos sensores (eixo dos sensores perpendicular ao eixo de rotação) e dos momentos de inércia do corpo.

Requisitos em atitude para o $3^{\circ}$ experimento (PPT, fase 2) - Aqui, o controle de atitude é necessário para garantir o apontamento adequado do vetor de impulso. Neste experimento, tem-se por objetivo verificar se o PPT é capaz de levantar a órbita do CubeSat, compensando o decaimento da altitude devido ao arrasto aerodinâmico. O propulsor tem alta frequência de disparo (praticamente contínuo) com duração de dias (ou semanas). A atitude precisa ser controlada continuamente durante o disparo. A estabilização por rotação prevista para a fase 1 pode ser repetida, mas uma manobra de atitude pode ser necessária, para apontar o eixo de rotação, que deve ser alinhado ao vetor de impulso, na direção do vetor velocidade, mantendo essa condição de apontamento. Uma precisão mínima na faixa de $5^{\circ}$ a $10^{\circ}$ foi estabelecida para o ângulo entre os vetores de impulso e velocidade, de modo que o experimento seja satisfatório.

\section{Proposta de ADCS Magnético para a Missão SERPENS II}

Sensores - A precisão exigida pelo propulsor, em termos de determinação de atitude (sensores), pode ser alcançada com o uso de magnetômetros (3 eixos), giroscópios (3 eixos) e sensor solar. Este conjunto compõe a proposta inicial.

Atuadores - Um sistema de controle magnético ativo de atitude é proposto para satisfazer os requisitos de estabilização, apontamento e manobra. Ele é constituído por três magnetorquers (configuração de 3 eixos) e circuito de eletrônica de potência para inversão de corrente e modulação. Magnetorquers podem ser obtidos com uma bobina de fio de cobre, capaz de gerar um dipolo magnético. Sua interação com o campo magnético da Terra gera o torque de controle.

Modos de operação do ADCS - O propulsor será posicionado no centro geométrico do 
CubeSat $3 \mathrm{U}$, centrado em $(0,0,0)$, com impulso apontado no sentido $+x$ (Figura 1-a). Os detectores de raios $\mathrm{X}$ e gama serão posicionados no corpo do satélite de maneira a terem seus campos de visada (CDV) apontados para as direções $+z$ e $-z$. Os experimentos deverão ser realizados em duas fases (Figura 1-b):

$\mathbf{1}^{\text {a }}$ fase - os experimentos 1 e 2 são efetuados. O satélite é estabilizado por rotação em torno do eixo $x$. O eixo de rotação deve ser controlado para manter-se apontando na direção zênitenadir. Essa configuração garante que os sensores não apontarão para a Terra e a rotação será útil na varredura da esfera celeste.

$2^{\mathbf{a}}$ fase - o experimento 3 (PPT) é realizado. O eixo $+x$ de rotação é manobrado em $90^{\circ}$ para alinhar-se na direção do vetor velocidade, sendo controlado para manter este apontamento durante todo o experimento. Os eixos $y$ e $z$ do corpo permanecerão girando no plano pitchyaw do sistema de coordenadas fixo na órbita.

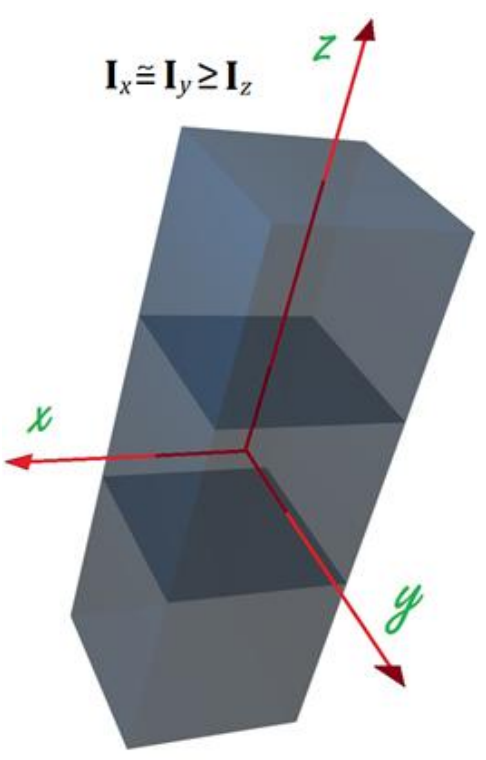

Figura 1-a: Sistema de coordenadas fixo no corpo.

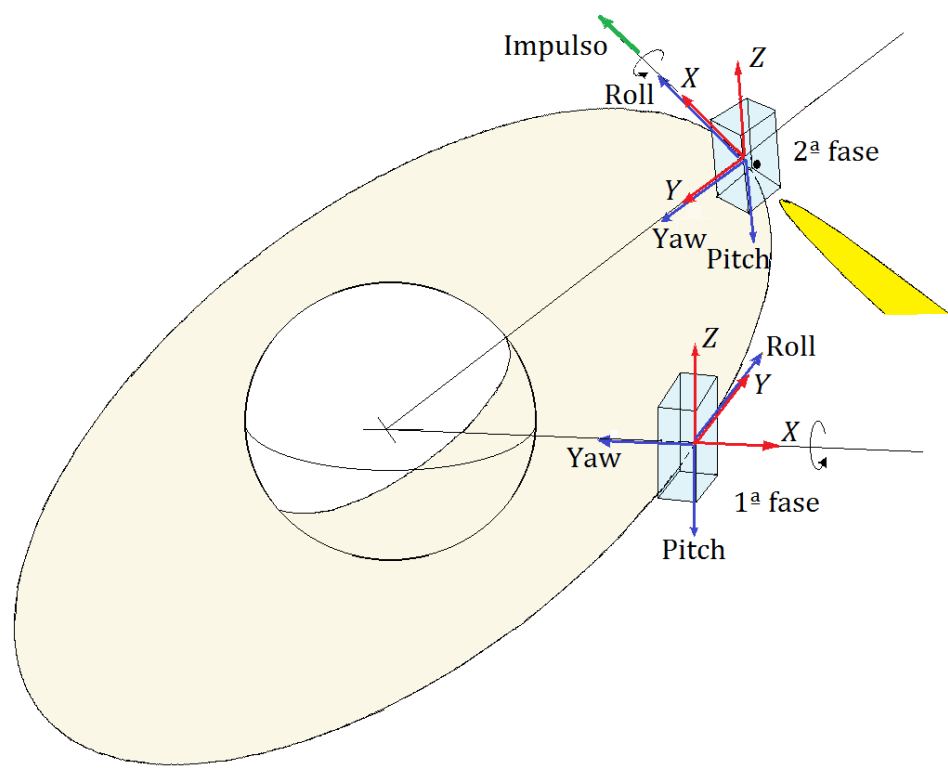

Figura 1-b: Fases dos experimentos e detalhes dos sistemas de coordenadas

Dentro das duas fases, ainda são previstos 5 modos de operação, que são descritos como: $1^{\circ}$ modo, descapotamento (detumbling): as velocidades angulares remanescentes são reduzidas. Movimentos de atitude podem ocorrer após o lançamento a partir do lançador espacial e também por causa de perturbações ambientais atuando no satélite. Esta ação de controle utilizará a lei de controle "Bdot" [10]. 20 modo, aquisição 1: depois do descapotamento, este modo será ativado para conduzir o CubeSat para a atitude correta para os experimentos 1 e 2, quando o satélite passa a girar em torno do eixo $x .3^{\circ}$ modo, controle 1: depois de adquirida a atitude adequada, este modo é ativado para mantê-la durante a realização dos experimentos 1 e 2. $4^{\mathbf{0}}$ modo, aquisição 2: após a realização dos experimentos 1 e 2, este modo conduzirá o CubeSat para a atitude adequada ao experimento 3. $\mathbf{5}^{\circ}$ modo, controle 2: depois de atingida a atitude adequada ao experimento do PPT, este modo deverá mantê-la durante o intervalo de ativação do mesmo. 


\section{Modelagens}

Um CubeSat 3U (Figura 1-a) é composto por três cubos alinhados com dimensões e peso padronizados [4]: eixo $z: 34,05 \mathrm{~cm}$; eixos $x$ e $y: 10 \mathrm{~cm} \mathrm{X} 10 \mathrm{~cm}$, com massa total menor que $3,9 \mathrm{~kg}$. O propulsor PPT tem dimensões $9 \times 9 \times 3 \mathrm{~cm}^{3}$ e massa menor que $280 \mathrm{~g} \mathrm{[3].}$ Considerando o propulsor, a matriz de inércia do satélite é calculada assumindo a matriz de inércia de um paralelepípedo simétrico e homogêneo com 3,6 kg, mais a matriz de inércia do propulsor, considerando-se este como uma placa central, com massa de $300 \mathrm{~g}$. Dessa forma, os momentos de inércia calculados resultam na matriz $\mathbf{I}_{\text {sat }}^{b}$ utilizada nas simulações, dada por $\mathbf{I}_{\text {sat }}^{b}=\operatorname{diag}(0,037 ; 0,034 ; 0,0032) \mathrm{kg} \cdot \mathrm{m}^{2}$.

Os sistemas de coordenadas utilizados são: sistema geocêntrico inercial $\left(O_{i} X_{1} X_{2} X_{3}\right.$; usaremos o J2000); sistema fixo no corpo do satélite $\left(O_{b} x_{1} x_{2} x_{3}\right.$; origem no centro de massa do satélite, eixos coincidentes com os eixos principais de inércia); sistema fixo na órbita ( $O_{o}$ rpy; origem no centro de massa do satélite e fixo na órbita nominal do satélite). Os eixos têm suas direções definidas na Figura 1-b. No caso em que os desvios em atitude são nulos, este sistema coincide com o sistema do corpo do satélite.

Equações do movimento - A equação (1) representa a dinâmica do satélite, descrevendo a velocidade angular de acordo com os torques atuantes $\mathbf{N}_{c}^{b}$ (torque de controle) e $\mathbf{N}_{\text {per }}^{b}$ (torques de perturbação) e a equação (2) representa a atitude por meio de quaternions $[2,8]$ :

$$
\begin{gathered}
\mathbf{I}_{s a t}^{b} \dot{\boldsymbol{\omega}}_{i b}^{b}+\boldsymbol{\omega}_{i b}^{b} \times \mathbf{I}_{s a t}^{b} \boldsymbol{\omega}_{i b}^{b}=\mathbf{N}_{c}^{b}+\mathbf{N}_{p e r}^{b} \\
\dot{\mathbf{q}}=\frac{1}{2} \boldsymbol{\Omega}\left(\omega_{o b}^{b}\right) \mathbf{q}
\end{gathered}
$$

onde q é o vetor de quaternions e $\boldsymbol{\Omega}\left(\omega_{o b}^{b}\right)$ é a matriz antissimétrica da velocidade angular.

A notação $\boldsymbol{\omega}_{i b}^{b}$ representa a velocidade angular (vetor) do sistema do corpo com relação ao sistema inercial (subscrito), escrita no sistema do corpo (sobrescrito). As velocidades angulares $\boldsymbol{\omega}_{i b}^{b}$ e $\boldsymbol{\omega}_{o b}^{b}$ são relacionadas por:

$$
\boldsymbol{\omega}_{i b}^{b}=\boldsymbol{\omega}_{o b}^{b}+\mathbf{R}_{o}^{b}\left[\begin{array}{lll}
0 & -\omega_{0} & 0
\end{array}\right]^{T}
$$

onde $\mathbf{R}_{o}^{b}$ é a matriz de rotação do sistema orbital para o sistema do corpo e $\omega_{0}$ é a velocidade angular orbital (constante, no caso de órbita circular).

Descapotamento (detumbling) - O objetivo do controle de descapotamento é gerar um momento magnético nas bobinas tal que a energia cinética de rotação do satélite seja dissipada. Este controle utiliza somente medidas do campo magnético terrestre.

O momento magnético $\mathbf{~ m}^{b}$ gerado nas bobinas é dado pela lei de controle $[1,8]$ :

$$
\mathbf{m}^{b}=-k \dot{\mathbf{B}}^{b}-\mathbf{m}_{c}
$$

onde $\dot{\mathbf{B}}^{b}$ é a variação do campo magnético terrestre sentido pelo satélite, $k$ é o ganho do controlador e $\mathbf{m}_{c}$ representa o momento magnético residual do satélite. Todas as variáveis estão representadas no sistema de coordenadas do corpo. Assim, o torque aplicado ao satélite pelas bobinas magnéticas $\mathbf{N}^{b}$ corresponde a: 


$$
\mathbf{N}^{b}=\left(-k \dot{\mathbf{B}}^{b}-\mathbf{m}_{c}\right) \times \mathbf{B}^{b}
$$

Simulação do descapotamento - A simulação do controle de descapotamento foi executada considerando as dimensões do satélite Serpens II descritas acima. O momento magnético máximo $m$ gerado por cada bobina é dado pela expressão $m=n i A$, onde $n$ é o número de espiras, $i$ é a corrente máxima e $A$ é a área da bobina. Nesta simulação, foram considerados $n$ $=250$ espiras, $i=0,1$ A e $A=0,006 \mathrm{~m}^{2}$, resultando em um momento magnético total $m=$ $0,15 \mathrm{Am}^{2}$, os quais são valores típicos para atuadores magnéticos de CubeSats $[1,2]$. O ganho do controlador foi ajustado em $k=10000$. O campo magnético terrestre pode ser modelado usando harmônicos esféricos de um potencial, cujos coeficientes são dados pelo modelo IGRF 2010 [6]. Porém, o modelo do campo magnético usado é o modelo de dipolo, que pode ser obtido calculando o modelo de harmônicos esféricos até o primeiro grau e todas as ordens. Este modelo apresenta boa precisão comparado com o modelo completo [5].

Dados o valor de $\mathbf{m}_{c}=\left[\begin{array}{lll}0 & 0 & 0,001\end{array}\right]^{T} \mathrm{Am}^{2}$ e a condição inicial de velocidade angular $\omega_{o b}^{b}=\left[\begin{array}{lll}15 & 15 & 15\end{array}\right]^{T} \%$ s, a Figura 3 mostra sua evolução à medida que o satélite avança na órbita. A Figura 4 mostra o momento magnético gerado pelas bobinas em cada eixo. A Figura 5 mostra os valores de cada variável dos quaternions.

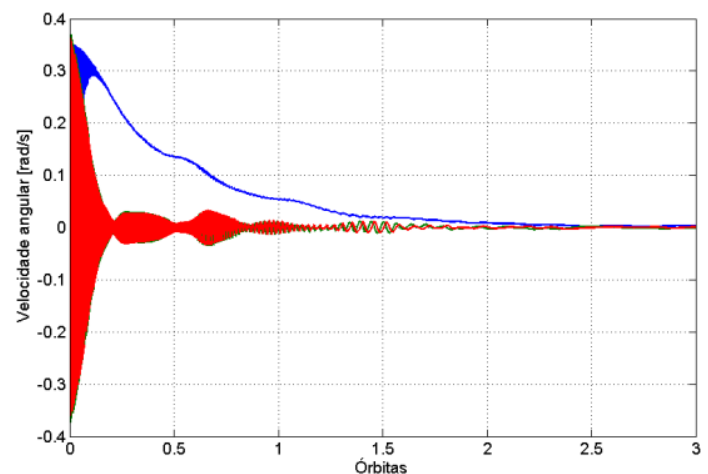

Figura 2: Velocidade angular do satélite.

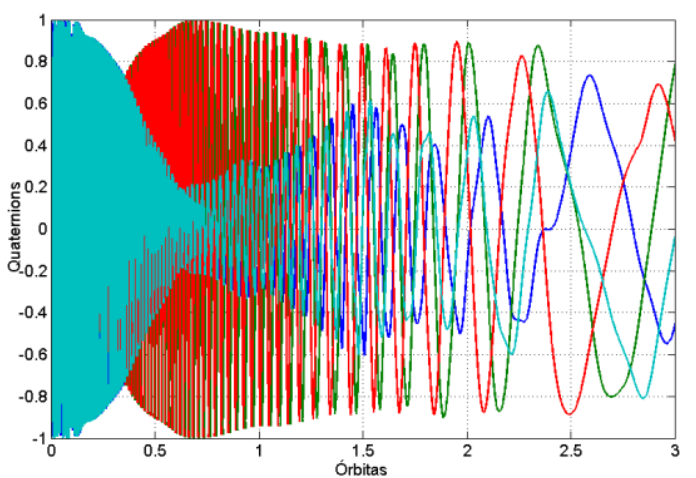

Figura 4: Evolução dos quaternions.

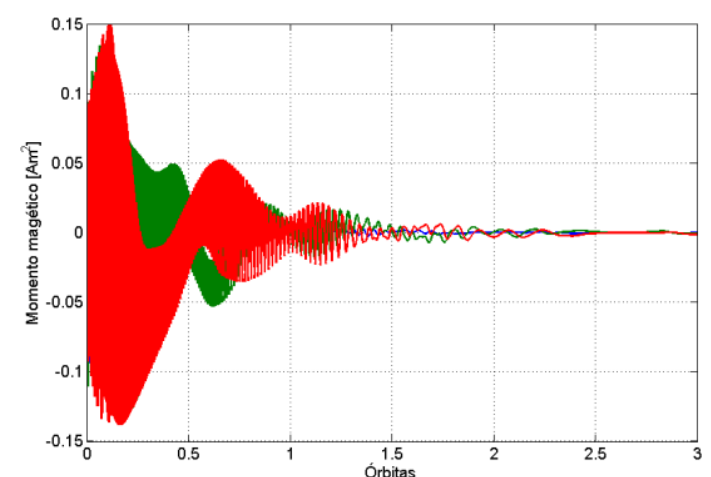

Figura 3: Momento magnético gerado por cada atuador.
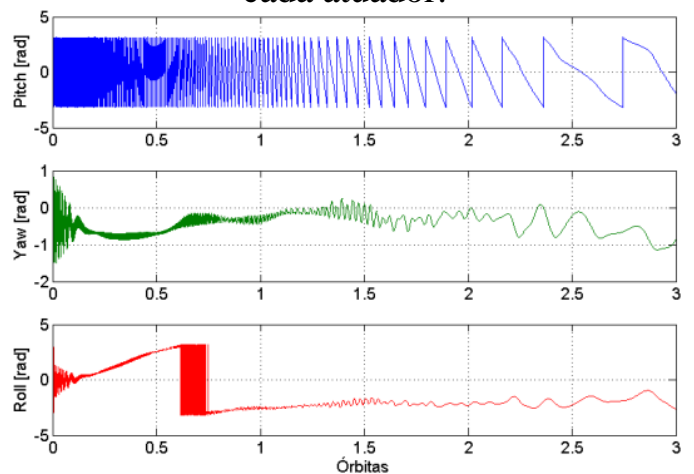

Figura 5: Ângulos de Euler do satélite.

Os gráficos mostram que o controle de descapotamento consegue dissipar a rotação do satélite dentro de três órbitas, mantendo a velocidade de rotação menor que $0,005 \mathrm{rad} / \mathrm{s}$ para as condições iniciais dadas. Assim, a orientação varia lentamente, como mostra o gráfico da Figura 5. Este gráfico mostra os ângulos de Euler calculados a partir dos quaternions. 


\section{Conclusões}

A missão SERPENS II foi apresentada de maneira geral e, mais especificamente, os requisitos em atitude da missão foram apresentados e discutidos.

Uma proposta de ADCS para a missão foi formulada e apresentada, em termos de hardware, software e da sua implementação (fases e modos de operação). As vantagens da proposta efetuada são a configuração exclusiva de ADCS para as duas fases da missão, juntamente com a simplicidade de hardware e software necessários para completar a missão (isso significa redução de custos, peso, tamanho e consumo de energia).

A modelagem do satélite e dos principais torques atuantes, em termos da sua dinâmica de atitude, foi efetuada. As simulações efetuadas para o primeiro modo de controle de atitude proposto, o descapotamento, mostraram resultados satisfatórios. Estes resultados mostraram a dissipação da velocidade angular inicial, levando o satélite a valores satisfatórios, da ordem de $0,003 \mathrm{rad} / \mathrm{s}$ em cada eixo, a partir dos quais os outros modos de controle podem atuar. Estes valores foram atingidos após 3 órbitas. Novas simulações dos modos adicionais deverão ser feitas no futuro para validar todos os modos da proposta efetuada.

\section{Referências}

[1] G. Arantes Jr., Estudo comparativo de técnicas de controle de atitude em 3 eixos para satélites artificiais. Instituto Nacional de Pesquisas Espaciais (Dissertação de mestrado), (2005).

[2] G. Bråthen, Design of Attitude Control System of a Double CubeSat. Norwegian University of Science and Technology (Master Thesis), (2013).

[3] Clyde Space. CubeSat plasma thruster (2015).

http://www.clyde-space.com/cubesat_shop/propulsion/303_cubesat-pulse-plasma-thruster

[4] Cubesat.org - CubeSat Acceptance Checklist (2015).

http://subdomain.cubesat.org/images/developers/3u_cac_rev12.pdf

[5] J. Davis, Mathematical Modeling of Earth's Magnetic Field. Technical Note, Virginia Tech, Blacksburg, VA, (2004).

[6] C. C. Finlay; S. Maus; C. D. Beggan, et al. International Geomagnetic Reference Field: the eleventh generation. Geophys. J. Int. 183, 2010, pp. 1216-1230, (2010), DOI: 10.1111/j.1365-246X.2010.04804.X

[7] D. T. Gerhardt and S. E. Palo, Passive magnetic attitude control for CubeSat spacecraft, Proceedings of the 24th Annual AIAA/USU Conference on Small Satellites. August 9-12, Logan, UT, USA, (2010).

[8] J. T. Gravdahl, Magnetic attitude control for satellites. In: Proc. of 43rd IEEE Conference on Decision and Control. IEEE, pp. 261-266, (2004), DOI: 10.1109/CDC.2004.1428640

[9] K. F. Jensen and K. Vinther, Attitude Determination and Control System for AAUSAT3. Aalborg University (Master Thesis), (2010).

[10] A. C. Stickler and K. T. Alfriend. Elementary Magnetic Attitude Control System. Journal of Spacecraft and Rockets, Vol. 13, No. 5 pp. 282-287, (1976), DOI: $10.2514 / 3.57089$ 